\title{
Assessment of oesophageal emptying in achalasia patients by intraluminal impedance monitoring
}

\author{
J. M. CONCHILlO, ${ }^{\star}$ M. SELIMAH, ${ }^{\star}$ A. J. BREDENOORD,$\dagger$ M. SAMSOM ${ }^{\star} \&$ A. J. P. M. SMOUT ${ }^{\star}$ \\ ${ }^{\star}$ Department of Gastroenterology and Hepatology, University Medical Center, Utrecht, The Netherlands \\ $\dagger$ Department of Gastroenterology, St Antonius Hospital, Nieuwegein, The Netherlands
}

\begin{abstract}
Oesophageal emptying can be assessed by radiographic and scintigraphic tests with radiation exposure or by multichannel intraluminal impedance monitoring (MII). The aim of this study was to evaluate the applicability of MII for the assessment of oesophageal emptying in achalasia patients. In 10 achalasia patients, impedance tracings were scored independently by three observers after ingestion of a 100-mL barium bolus. Bolus clearance time (BCT) and height of barium column were scored using fluoroscopic images acquired at 20-s intervals. All patients showed a low baseline impedance level in the distal oesophagus. Air trapping in the proximal oesophagus was detected in nine patients. BCT on MII was similar to that on fluoroscopy in $40-70 \%$ of the patients. Correlations between height of barium on fluoroscopy and fluid level on MII were poor to moderate at different time intervals. Concordance (Kendall's coefficient) between the three observers for assessment of fluid level on MII was $0.31(P=0.04)$ at 1 and $5 \mathrm{~min}$, $0.26(P=0.08)$ at 10 and $0.44(P=0.01)$ at $15 \mathrm{~min}$. We conclude that in achalasia patients, low baseline impedance levels and air entrapment in the proximal oesophagus limit the value of intraluminal impedance monitoring as a test of oesophageal emptying.
\end{abstract}

Keywords achalasia, impedance monitoring, oesophageal emptying.

\section{INTRODUCTION}

Idiopathic achalasia is an inflammatory disease of unknown aetiology characterized by oesophageal aper-

Address for correspondence

José M. Conchillo, MD, Department of Gastroenterology and Hepatology, Erasmus Medical Center, PO Box 2040, 3000 CA Rotterdam, The Netherlands.

Tel: +31 10 4635942; fax: +31 104365916 ;

e-mail: jconchillo@hotmail.com

Received: 15 February 2006

Accepted for publication: 2 May 2006 istalsis and failure of lower oesophageal sphincter (LOS) relaxation due to loss of inhibitory nitrinergic neurons in the oesophageal myenteric plexus. ${ }^{1}$ Dysphagia, chest pain and regurgitation are the main symptoms in patients with achalasia.

Manometric patterns and LOS pressure in particular are the most common follow-up parameters in achalasia patients. ${ }^{2}$ However, as in other oesophageal motor disorders it is often difficult to correlate symptoms with objective manometric or endoscopic patterns. At present, conclusions about the efficacy of oesophageal motor activity are indirect inferences based upon experience gathered from previous studies of combined manometry and radiology ${ }^{3}$ or radionuclide scintigraphy. ${ }^{4}$ Such studies are logistically complex and cumbersome and not readily applied to routine clinical practice. In addition, the effect of therapy in achalasia patients is often difficult to objectify and there is a need for an objective and simple test during the followup period after treatment. The radionuclide oesophageal transit using a solid meal has been found to be a useful study to quantitate results of therapy in achalasia. ${ }^{5,6}$ Two studies showed that the upright timed barium oesophagram is an objective test in assessing oesophageal emptying ${ }^{7}$ and evaluating the success of pneumatic dilation for achalasia. ${ }^{8}$

Recently, intraluminal impedance measurement has been introduced as a technique to investigate bolus transport in the oesophagus and duodenum. ${ }^{9-15}$ Imam et al. ${ }^{16}$ validated the impedance technique in healthy subjects by tracking the movement of a swallowed barium bolus with simultaneous fluoroscopy and impedance monitoring. A strong correlation was found between the two methods and the authors concluded that impedance was able to determine accurately the timing of oesophageal lumen filling and emptying. This method can be easily implemented in clinical practice and it has no radiation hazards, in contrast to radiological and scintigraphic examinations.

However, application of the impedance measurement technique in patients with achalasia was found 
to be associated with technical problems. Due to low base impedance values and air accumulation (leading to high impedance) it can be difficult to track the transit of the traditional $5-10 \mathrm{~mL}$ saline boluses in achalasia patients. ${ }^{17-19}$

The aim of this study was therefore to evaluate the applicability of intraluminal impedance measurement for the assessment of oesophageal emptying in achalasia patients, by concurrent videofluoroscopy and impedance recording during the oesophageal clearance of a barium bolus. In particular, we wished to explore the possibility that the height of the fluid column in the oesophagus could be measured with the impedance technique.

\section{MATERIALS AND METHODS}

\section{Subjects}

Ten achalasia patients from our outpatient clinic (six men and four women, mean age 44 years, range 20-64) were included in the study. The diagnosis of achalasia was made according to established manometric criteria. Written informed consent was obtained from all the patients and the protocol was approved by the Medical Ethics Committee of the University Medical Center Utrecht.

\section{Oesophageal impedance recording}

Intraluminal electrical impedance was recorded with a 2.3-mm diameter polyvinyl assembly containing a series of cylindrical electrodes, each $4 \mathrm{~mm}$ in axial length and spaced at 2-cm intervals, except for the most proximal one (segment 1) which was spaced at 1-cm interval from segment 2, corresponding to one recording segment (Aachen University of Technology, FEMU, Aachen, Germany). The assembly contained a total of seven measuring segments and each segment was connected to an impedance measurement device that delivered a measuring current of $<6 \mathrm{~mA}$ at a frequency of $2 \mathrm{kHz}$. The most distal recording segment (segment 7) was located at the level of manometrically localized LOS and the other impedance segments (segments 6-1) were located at 0-2, 2-4, 4-6, 8-10, 12-14 and 15-17 $\mathrm{cm}$ above the upper border of the LOS (Fig. 1). Impedance signals were recorded in a digital system (Aachen University of Technology) using a sample frequency of $50 \mathrm{~Hz} .^{15}$

\section{Videofluoroscopy}

In this study, the patient was sitting in upright position in front of a videofluoroscopy device (Easy Diagnost,

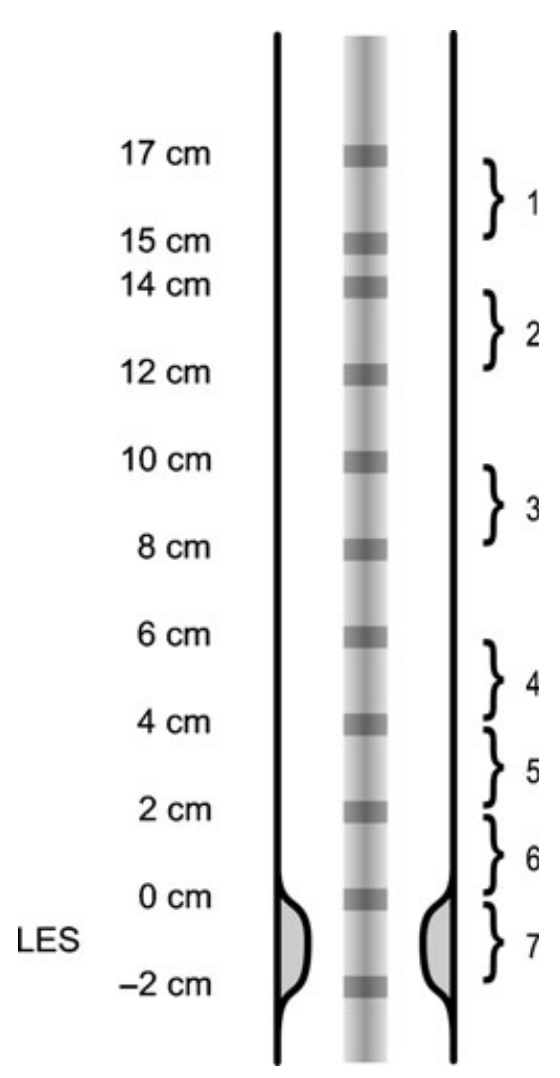

Figure 1 Position of the impedance catheter in the oesophagus with respect to the manometric upper border of the lower oesophageal sphincter (LOS).

Philips Medical Systems, Best, The Netherlands). The $\mathrm{X}$-ray images were online digitized during the investigations using an image acquisition card (MMS Image Digitizing System, MMS, Enschede, The Netherlands) and saved on disk for further analysis.

\section{Study protocol}

The study was performed by recording simultaneously videofluoroscopy and impedance during the oesophageal clearance of a $100-\mathrm{cm}^{3}$ barium swallow. Three days before the study, patients discontinued drugs that influence gastrointestinal motility. On the day before the study patients used a liquid diet and on the day of the study they were fasted for at least $8 \mathrm{~h}$. The impedance catheter was introduced into the oesophagus via an anaesthetized nostril and the most distal segment (segment 7) was positioned at the level of the LOS, as determined during a recent oesophageal manometry. At the start of the impedance recording a baseline fluoroscopic image was recorded and at that moment a marker was put on the impedance datalogger. The location of segment 7 at the level of the LOS 
was checked fluoroscopically and corrected when required. After $1 \mathrm{~min}$ the patient was instructed to drink $100 \mathrm{~cm}^{3}$ of barium suspension (Liquid Polibar $1 \mathrm{~g} \mathrm{~mL}^{-1}$, E-Z-EM Canada Inc., Montreal, Canada). From this moment onwards impedance monitoring was recorded continuously during $15 \mathrm{~min}$ and a fluoroscopic image was recorded every $20 \mathrm{~s}$. Every time a fluoroscopic image was made, the event marker on the impedance datalogger was pushed. Recording was stopped when oesophageal emptying was reached or when bolus clearance (judged fluoroscopically) had not taken place after $15 \mathrm{~min}$.

\section{Data analysis}

The fluoroscopic images of every patient were scored for bolus clearance time (BCT) which was defined as the time when barium column passed the impedance segment just above the LOS (segment 6). Moreover, the barium column level was analysed at 1, 5, 10 and 15 min by recognizing the impedance channel rings where the barium column was located.

The impedance tracings were analysed independently by three observers (JMC, MS and AJB). Each observer was blinded for the results of the fluoroscopic evaluation. BCT was scored as the time until recovery of the impedance to $50 \%$ of the baseline value, at segment $6(2 \mathrm{~cm}$ above LOS). The height of the fluid column, as inferred from the impedance signals was scored at 1, 5, 10 and $15 \mathrm{~min}$, and expressed as impedance segment number.

\section{Statistical analysis}

The data were expressed as mean ( \pm SEM). Spearman's correlation coefficient was calculated to express the correlation between videofluoroscopy and impedance monitoring. Interobserver correlation for fluid level on impedance monitoring was tested using Kendall's coefficient of concordance $(W) . P$-value $<0.05$ was accepted as indicating statistical significance. All statistical analyses were performed using commercially available computer software (SPSS 11.5 for Microsoft Windows, Chicago, IL, USA).

\section{RESULTS}

\section{Patient characteristics}

The mean $( \pm$ SEM) duration of disease was 7.6 $( \pm 2.1)$ years. None of the patients underwent myotomy or botulinum treatment in the past. During the 12 months before the study, pneumatic dilation was performed in seven of 10 the patients. The mean basal and nadir LOS pressure during swallows were $14( \pm 2)$ and $10( \pm 2) \mathrm{mmHg}$, respectively. The mean distal oesophageal contraction amplitude was $19( \pm 4) \mathrm{mmHg}$. Manometric parameters of treated and untreated patients were similar.

\section{Impedance characteristics}

All patients showed a low baseline impedance level $(<1200 \Omega)$ in the distal oesophagus, suggesting stasis of fluids. In contrast, no signs of stasis were found in any of the blank oesophagrams at the start of the test. Three of the 10 patients showed at least one period of regurgitation and in nine patients pathological air presence within the proximal oesophagus (air trapping) was detected. In Fig. 2, an example of air trapping on impedance and fluoroscopy is shown.

\section{Bolus clearance time on videofluoroscopy and impedance monitoring}

An example of bolus clearance on impedance and videofluoroscopy is shown in Fig. 3. As shown in Table 1, there were considerable differences in BCT on impedance monitoring as scored by the three observers. Two observers scored BCT on impedance similarly $( \pm 1 \mathrm{~min})$ to the time on videofluoroscopy in $70 \%$ of the patients while the third observer found a similar clearance time in $40 \%$ of the patients.

On videofluoroscopy, $80 \%$ of the patients showed a BCT longer than $15 \mathrm{~min}$. On impedance monitoring, the percentage of patients having a BCT longer than $15 \mathrm{~min}$ as scored by the three observers was $50 \%, 40 \%$ and $20 \%$ respectively (mean $37 \%$ ).

In only three of the 10 patients all the three observers found on impedance monitoring a BCT similar to the time on videofluoroscopy. Concordance between two observers was achieved in five patients.

\section{Barium column level on videofluoroscopy and fluid level on impedance monitoring}

As shown in Fig. 4, the correlation between the height of barium column on fluoroscopy and the fluid level on impedance was poor to moderate. A significant, albeit weak, correlation was found at $10 \mathrm{~min}(r=0.65$, $P<0.05)$ and $15 \min (r=0.63, P=0.05)$.

Kendall's coefficient of concordance $(W)$ between the three observers for the fluid level on impedance monitoring was $0.31(P=0.04)$ at 1 and $5 \mathrm{~min}, 0.26$ $(P=0.08)$ at $10 \mathrm{~min}$ and $0.44(P=0.01)$ at $15 \mathrm{~min}$. 


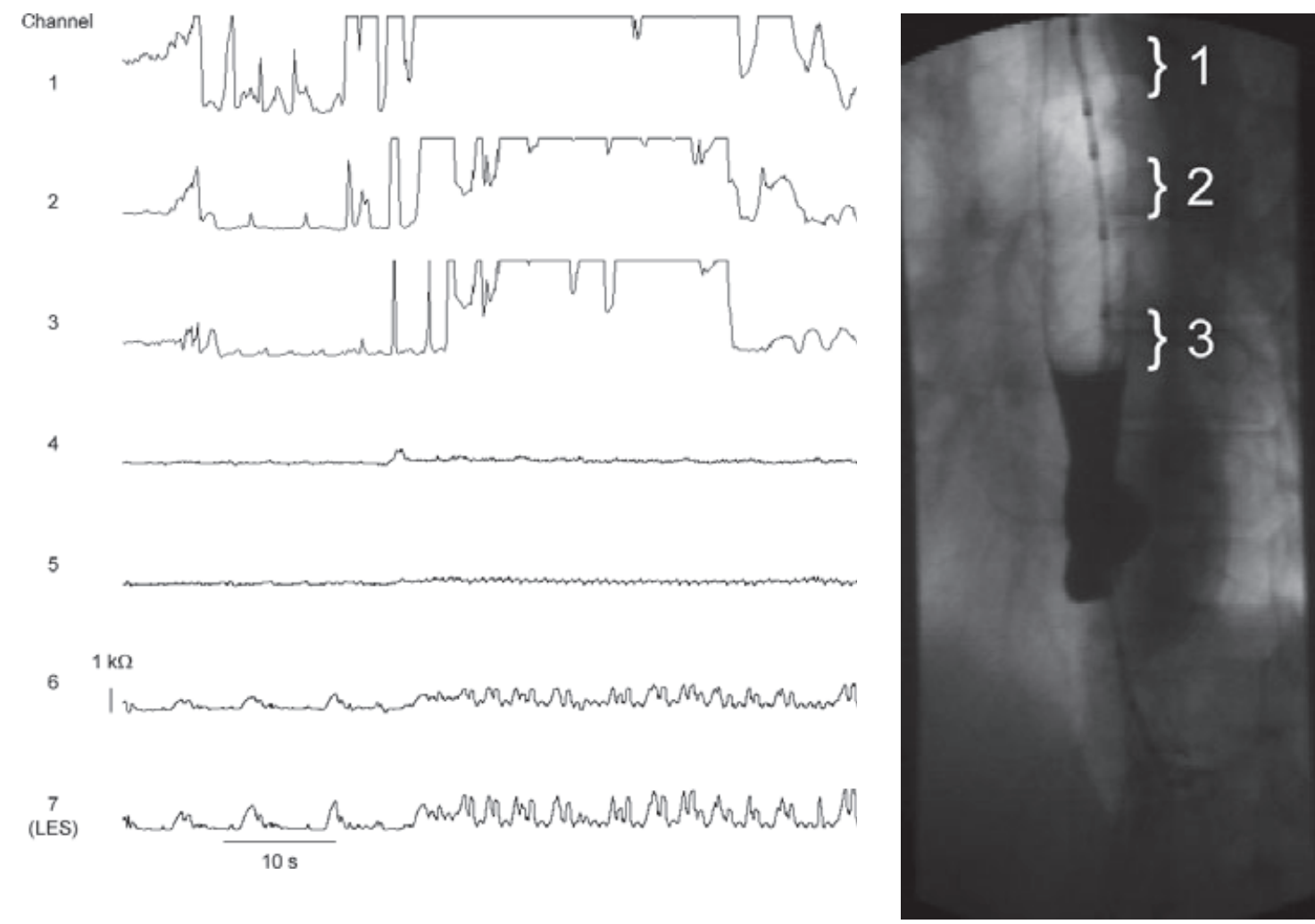

Figure 2 Example of pathological air presence within the proximal oesophagus (air trapping) on impedance monitoring (high impedance levels) and fluoroscopy.

\section{DISCUSSION}

In the present study, assessment of oesophageal emptying in achalasia patients by intraluminal impedance monitoring was compared to fluoroscopy, which is considered to be an objective test for this purpose. Compared to fluoroscopy, the impedance technique has been validated for oesophageal emptying in healthy volunteers and in a recent study, impedance and videoesophagram were found to have excellent correlation in identifying bolus transit patterns in healthy subjects. ${ }^{16}$ Theoretically, the impedance technique could substitute the timed barium oesophagram and the radionuclide oesophageal transit measurement in the follow-up and in assessing the success of treatment in achalasia patients; however, this had not been studied yet.

Three previous studies have explored the applications of oesophageal impedance monitoring in patients with achalasia. Nguyen et al. ${ }^{17}$ described low baseline impedance levels, regurgitation of luminal contents and air presence within the proximal oesophagus (air trapping) during swallowing. A previous study from our lab, in which 40 patients with non-obstructive dysphagia were evaluated with the impedance technique, showed that most of the swallows in the three achalasia patients could not be quantified due to low preswallow impedance baseline and almost all swallows had abnormal transit patterns. ${ }^{18}$ Using simultaneous manometry and impedance monitoring in a group of patients with different manometric diagnoses, Tutuian et al. described abnormal bolus transit among all achalasia patients, as compared to established impedance values in healthy volunteers. ${ }^{19}$ However, in the three studies mentioned no attempt was made to correlate the transit and emptying patterns observed with impedance monitoring with those obtained with another test, such as fluoroscopy. Furthermore, the impedance monitoring technique had not yet been used to estimate the height of the fluid column in the oesophagus of achalasia patients.

Air accumulation within the proximal oesophagus was more frequently present in the tracings of our patients than in Nguyen's study ${ }^{17}$ and this pattern hampered precise assessment of bolus clearance. The higher incidence of air trapping in our study could be explained by longer stasis of fluids in the oesophagus 

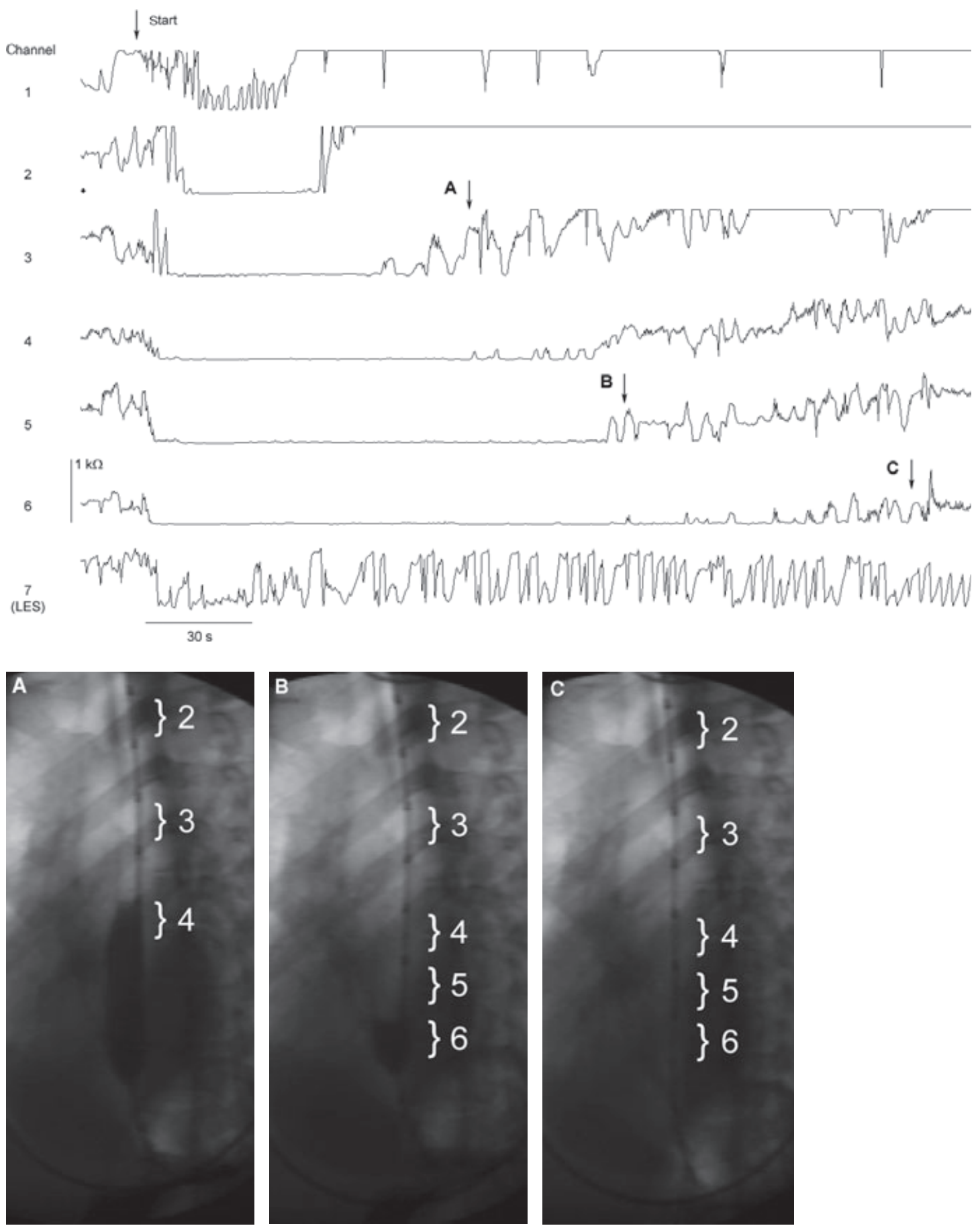

Figure 3 Bolus transit in patient 10 on impedance and fluoroscopy. (A, B) Bolus clearance at levels 3 and 5 (2.00 and 2.40 min respectively). (C) Bolus clearance $2 \mathrm{~cm}$ above lower oesophageal sphincter (LOS) (level 6, $3.40 \mathrm{~min}$ ). Note air accumulation within the three proximal channels in the second part of the tracing.

due to the use of a higher bolus volume $(100 \mathrm{~mL})$ and different viscosity (barium suspension) than in Nguyen's study (eight liquid and semisolid boluses of $10 \mathrm{~mL})$.
Assessment of BCT on the basis of impedance monitoring by the three observers was poor, when compared to BCT on fluoroscopy. There was a tendency by the observers to score a faster BCT than 
Table 1 Bolus clearance time (min) on videofluoroscopy and on impedance monitoring as scored by the three observers

Impedance

Patient Videofluoroscopy Observer 1 Observer 2 Observer 3

\begin{tabular}{llccc}
\hline 1 & $>15$ & $>15$ & 14.50 & $>15$ \\
2 & $>15$ & 2.45 & $>15$ & 1.10 \\
3 & $>15$ & 10.15 & $>15$ & 2.00 \\
4 & 2.40 & 1.45 & 13.05 & 3.00 \\
5 & $>15$ & 8.30 & 8.35 & 0.50 \\
6 & $>15$ & $>15$ & $>15$ & $>15$ \\
7 & $>15$ & $>15$ & 14.45 & 1.00 \\
8 & $>15$ & $>15$ & $>15$ & 2.40 \\
9 & $>15$ & $>15$ & 8.50 & 0.50 \\
10 & 3.40 & 3.50 & 3.45 & 2.30 \\
\hline
\end{tabular}

with fluoroscopy, possibly due to temporary impedance rises caused by air trapping. Despite the observers' experience with analysis of impedance tracing, the correlation between impedance monitoring and fluoroscopy for the assessment of the height of barium column was found to be moderate to poor because of the pathological bolus transport patterns discussed before. Concordance between observers regarding BCT and height of fluid level on impedance monitoring was moderate.

In our study we used $100 \mathrm{~mL}$ of barium for the assessment of oesophageal emptying, as this is a frequently used volume for this purpose. ${ }^{8}$ Although this volume was satisfactory for the fluoroscopic assessment, it led to more air trapping and delayed bolus emptying on impedance monitoring. A test protocol with multiple low-volume barium boluses given after sufficiently long-time intervals could improve the performance of the test.

In summary, interpretation of oesophageal emptying in achalasia patients by impedance monitoring is difficult due to a combination of abnormal patterns such as low baseline impedance levels, regurgitation of luminal content and air entrapment in the proximal oesophagus. It is therefore felt to be unlikely that assessment of oesophageal emptying by impedance monitoring will prove to be a clinically useful tool in the management of achalasia patients.
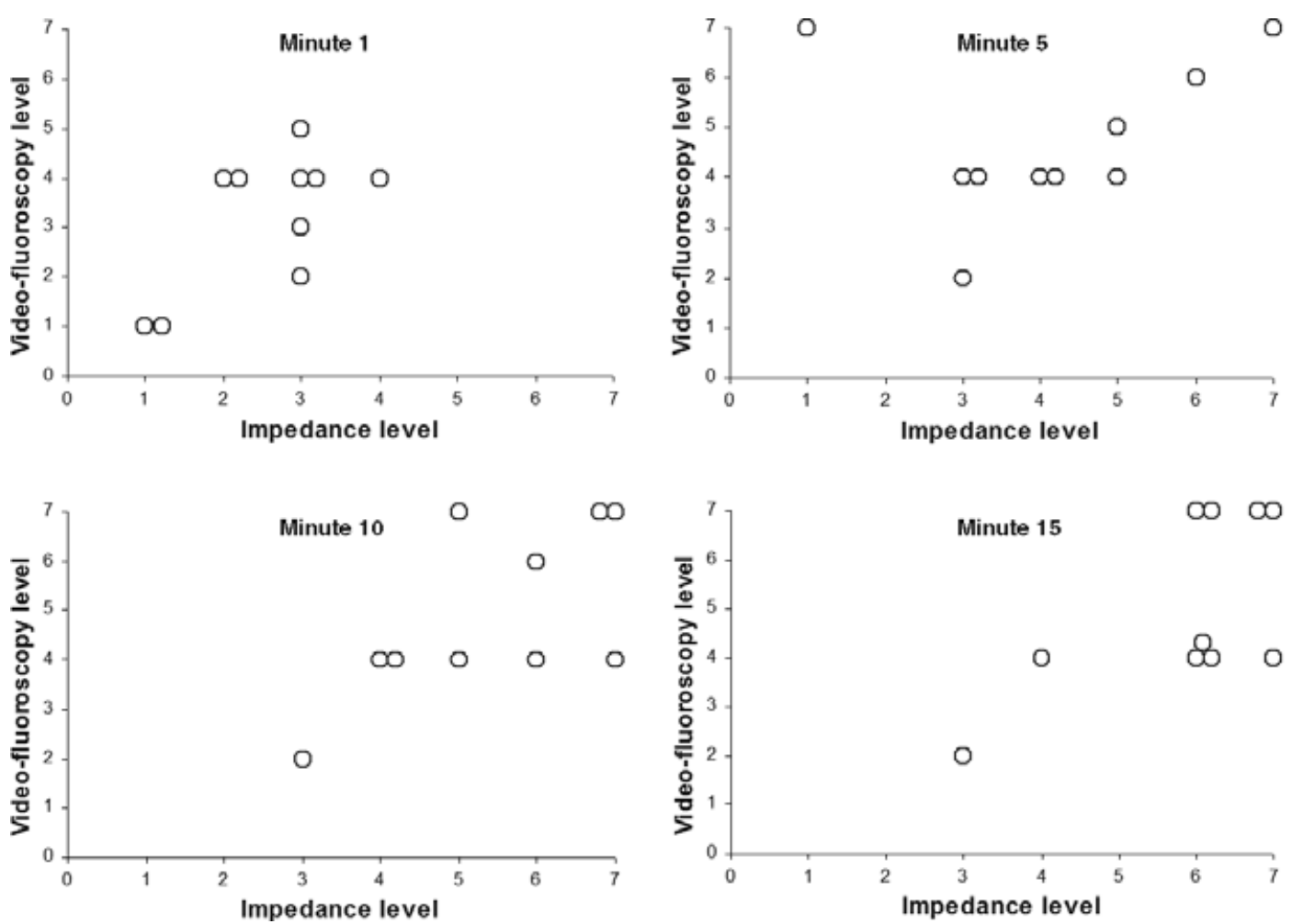

Figure 4 Correlation between videofluoroscopy and impedance monitoring (median value of the three observers) regarding barium column levels at $1,5,10$ and $15 \mathrm{~min}$. Minute $1: r=0.53, P=0.11$; minute $5: r=0.42, P=0.22 ;$ minute $10: r=0.65, P=0.04$; minute 15: $r=0.63, P=0.05$ (Spearman test). 


\section{REFERENCES}

1 Park W, Vaezi F. Etiology and pathogenesis of achalasia: the current understanding. Am J Gastroenterol 2005; 100: 1404-14.

2 Eckardt VF, Aignheer C, Bernhard G. Predictors of outcome in patients with achalasia treated by pneumatic dilation. Gastroenterology 1992; 103: 1732-8.

3 Kahrilas PJ, Dodds WJ, Hogan WJ. Effect of peristaltic dysfunction on esophageal volume clearance. Gastroenterology 1988; 94: 73-80.

4 Richter JE, Blackwell JN, Wu WC, Johns DN, Cowan RJ, Castell DO. Relationship of radionuclide liquid bolus transport and esophageal manometry. I Lab Clin Med 1987; 109: 217-24.

5 Holloway RH, Krosin G, Lange RC, Baue AE, McCallum RW. Radionuclide esophageal emptying of a solid meal to quantitate results of therapy in achalasia. Gastroenterology 1983; 84: 771-6.

6 Shen YY, Shiau YC, Sun SS, Kao CH. Using radionuclide esophageal emptying test to evaluate pneumatic dilation effects for achalasia. Hepatogastroenterology 2001; 48: 1061-3.

7 De Oliveira JM, Birgisson S, Doinoff C et al. Timed barium swallow: a simple technique for evaluating esophageal emptying in patients with achalasia. Am J Roentgenol 1997; 169: 473-9.

8 Vaezi MF, Baker ME, Richter JE. Assessment of esophageal emptying post pneumatic dilation: use of timed barium esophagram. Am J Gastroenterol 1999; 94: 1802-7.

9 Silny J. Intraluminal multiple impedance procedure for measurement of gastrointestinal motility. I Gastrointest Motil 1991; 3: 151-62.

10 Nguyen HN, Silny J, Wuller S, Marschall HU, Rau G, Matern S. Chyme transport patterns in human duodenum, determined by multiple intraluminal impedancometry. Am I Physiol 1995; 268: G700-8.
11 Frieling T, Hermann S, Kuhlbusch R et al. Comparison between intraluminal electric impedance measurement and manometry in the human esophagus. Neurogastroenterol Motil 1996; 8: 45-50.

12 Nguyen HN, Silny J, Matern S. Multiple intraluminal electrical impedancometry for recording of upper gastrointestinal motility: current results and further implications. Am J Gastroenterol 1999; 94: 306-17.

13 Tutuian R, Vela MF, Balaji NS, et al. Esophageal function testing with combined multichannel intraluminal impedance and manometry: multicenter study in healthy volunteers. Clin Gastroenterol Hepatol 2003; 1: 174-82.

14 Simren M, Silny J, Holloway R, Tack J, Janssens J, Sifrim D. Relevance of ineffective esophageal motility during esophageal acid clearance. Gut 2003; 52: 784-90.

15 Bredenoord AJ, Weusten BL, Timmer R, Smout AJ. Minimum sample frequency for multichannel intraluminal impedance measurement of the esophagus. Neurogastroenterol Motil 2004; 16: 713-9.

16 Imam H, Shay S, Ali A, Baker M. Bolus transit patterns in healthy subjects: a study using simultaneous impedance monitoring, videoesophagram, and esophageal manometry. Am I Physiol Gastrointest Liver Physiol 2005; 288: G1000-6.

17 Nguyen HN, Domingues GR, Winograd R, Lammert F, Silny J, Matern S. Impedance characteristics of esophageal motor function in achalasia. Dis Esophagus 2004; 17: 4450 .

18 Conchillo JM, Nguyen NQ, Samsom M, Holloway RH, Smout AJ. Multichannel intraluminal impedance monitoring in the evaluation of patients with non-obstructive dysphagia. Am J Gastroenterol 2005; 100: 2624-32.

19 Tutuian R, Castell DO. Combined multichannel intraluminal impedance and manometry clarifies esophageal function abnormalities: study in 350 patients. Am I Gastroenterol 2004; 5: 1011-9. 